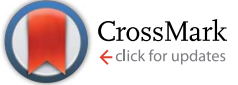

Cite this: Chem. Sci., 2015, 6, 6583

\title{
Light driven mesoscale assembly of a coordination polymeric gelator into flowers and stars with distinct properties $\uparrow$
}

\author{
Rahul Dev Mukhopadhyay, ${ }^{\text {ab }}$ Vakayil K. Praveen, ${ }^{a}$ Arpan Hazra, ${ }^{c}$ Tapas Kumar Majic \\ and Ayyappanpillai Ajayaghosh*ab
}

Control over the self-assembly process of porous organic-inorganic hybrids often leads to unprecedented polymorphism and properties. Herein we demonstrate how light can be a powerful tool to intervene in the kinetically controlled mesoscale self-assembly of a coordination polymeric gelator. Ultraviolet light induced coordination modulation via photoisomerisation of an azobenzene based dicarboxylate linker followed by aggregation mediated crystal growth resulted in two distinct morphological forms (flowers and stars), which show subtle differences in their physical properties.

Received 20th June 2015

Accepted 6th August 2015

DOI: $10.1039 / \mathrm{c} 5 \mathrm{sc0} 02233 a$

www.rsc.org/chemicalscience

possibility of light sensitive metal-organic systems. ${ }^{4}$ In this

\section{Introduction}

Mesoscopic science is broadly defined as the space between nanoscopic (atoms, molecules and assemblies up to a few nanometers in size) and the macroscopic (hierarchical assemblies, few micrometers in size or more) domains. The evolution of mesoscopic materials is controlled by chemical and physical processes, wherein atoms and molecules interact through physical forces, guided by thermodynamic and kinetic phenomena. Molecular self-assembly is a key nucleation point of mesoscopic evolution of functional materials and occupies a unique space in the field of 'nanoarchitectonics', ${ }^{1}$ which is a novel conceptual idea for the construction of functional materials via control over reactions or assembly of molecules making use of different physical stimuli. ${ }^{1 c}$ In recent years, metalorganic frameworks (MOFs) or porous coordination polymers (PCPs), ${ }^{2,3}$ known for their highly crystalline porous nature and ubiquitous applications have been receiving attention in the field of nanoarchitectonics. While wide range applications are envisaged for MOFs and PCPs, reversible control on the porosity and gas adsorption properties of such materials remain a challenging task. Therefore, an innovative approach in tuning the properties of MOFs or PCPs is of paramount importance. Such considerations encourage chemists to explore the

${ }^{a}$ Photosciences and Photonics Group, Chemical Sciences and Technology Division, India.E-mail: ajayaghosh@niist.res.in

${ }^{b}$ Academy of Scientific and Innovative Research (ACSIR), CSIR-National Institute for Interdisciplinary Science and Technology (CSIR-NIIST), Trivandrum-695019, India

${ }^{c}$ Chemistry and Physics of Materials Unit, Jawaharlal Nehru Centre for Advanced Scientific Research (JNCASR), Jakkur, Bangalore 560064, India

$\dagger$ Electronic supplementary information (ESI) available: Text, figures, and tables depicting different synthetic procedures, detailed experimental procedures, characterisation data including FT-IR, microscopic analyses, TGA, EDAX and SAED analyses, pore size distribution. See DOI: 10.1039/c5sc02233a context, azobenzene, a widely explored photoswitch, ${ }^{5}$ has been utilised recently by a number of research groups to design photoresponsive crystalline porous materials. ${ }^{6}$ However, photoisomerisation of directly linked azobenzene moieties in a rigid coordination framework is often found to be difficult. ${ }^{4 b}$ Therefore, an alternate approach is required to explore the utility of cis-trans photoisomerisation of azobenzene moieties in a metal-organic coordination framework.

Unlike rigid crystalline MOFs or PCPs, amorphous organogels are an entirely different class of soft material possessing a flexible supramolecular network. ${ }^{7-10}$ Gels are known to provide an excellent medium to create a dynamic environment, ideal for crystal growth process via defect rectification during reversible self-assembly. ${ }^{11}$ A gel medium is also a clever option to create hierarchical mesoscopic structures having complex forms. ${ }^{12}$ While there are several reports on photoresponsive gels, ${ }^{5 c, 7,8 e, 13}$ reports on photoresponsive microporous coordination polymeric gels are rare. ${ }^{\mathbf{1 4}}$ Moreover, a deeper understanding of the structural evolution of MOFs and PCPs at the mesoscopic scale under the influence of light is, as yet, unknown. This is rather surprising when considering the importance of mesoscopic structures in the field of materials science. ${ }^{15}$ Herein, we combine the chemistry of metal-organic materials and gels to create photoresponsive structures, to understand the effect of light on the kinetic pathways of mesoscopic structural evolution in synthetic molecular systems and study their properties. For this purpose, we designed a photoresponsive coordination polymeric gelator, which forms a gel having a flower-like morphology. Interestingly, pre-synthetic photoirradiation of the gelator resulted in star-shaped morphology with distinct rheological and gas adsorption properties. 


\section{Results and discussion}

\section{Synthesis and characterization of coordination polymeric gels CPG1 and CPG2}

Our experience with supramolecular gels, ${ }^{8 e}$ encouraged us to exploit the Materials of Institute Lavoisier (MIL) family of coordination polymers, ${ }^{\mathbf{1 6}}$ many of which are known for instantaneous gelation of the reaction mixture. Since the structural rigidity of MOFs and PCPs do not facilitate post-synthetic photoisomerisation of azobenzene on larger length scales, ${ }^{4 \boldsymbol{b}, \boldsymbol{6} \boldsymbol{d}}$ we hypothesised that if a pre-synthetic isomerisation is carried out, the cis form of azobenzene should in principle get locked in the rigid matrix of the hybrid material thereby leading towards self-assembled forms with different properties. In a typical experiment a gently warmed mixture of the azobenzene-4, $4^{\prime}$ dicarboxylic acid (ADA) and triethylamine in dimethyl sulfoxide (DMSO) was added to an equimolar quantity of $\mathrm{Fe}\left(\mathrm{NO}_{3}\right)_{3} \cdot 9 \mathrm{H}_{2} \mathrm{O}$ in same volume of DMSO, leading to immediate gelation (Fig. 1). Gelation was observed in polar solvents such as DMSO and dimethyl formamide (DMF) at a critical gelator concentration (CGC) of $20 \mathrm{mg} \mathrm{mL}^{-1}\left(7.4 \times 10^{-2} \mathrm{M}\right)$ with respect to ADA and was found to be highly sensitive towards mechanical disturbance. ${ }^{8 a}$ However, the gel was not thermoreversible in nature by virtue of its coordination network.

The involvement of metal-ligand coordination responsible for such gelation was confirmed from Fourier-transform infrared (FT-IR) spectral studies. ${ }^{17}$ The intense IR band at $1688 \mathrm{~cm}^{-1}$ corresponding to asymmetric carbonyl stretching frequency of the free acid ADA diminishes with considerable broadening in the case of coordination polymeric gel (CPG1), with the appearance of a new band at $1413 \mathrm{~cm}^{-1}$, corresponding to the symmetric stretching frequency. ${ }^{17 b}$ Deconvolution of the broad band at $\sim 1700 \mathrm{~cm}^{-1}$ suggests that the separation between the asymmetric and symmetric carbonyl stretching frequency increases in CPG1 when compared to ADA, as evidenced from the higher wavenumber peak contributions at 1700,1717 and $1731 \mathrm{~cm}^{-1}$. This observation primarily suggests the presence of unidentate coordination in CPG1 although the presence of bidentate binding modes cannot be completely ruled out as indicated by the peak contribution at $1671 \mathrm{~cm}^{-1}$ (Fig. $\mathrm{S} 1 \dagger) .{ }^{17 a}$

Scanning electron microscopy (SEM) analysis of the aforesaid gel revealed the formation of macroscopic sheets (Fig. 2). Furthermore, these sheets have a tendency to stack one above the other to form various metastable superstructures (Fig. S2 $\dagger$ ). Detailed morphological analysis of the gel sample by SEM showed the formation of the metal-organic gel through an evolutionary morphogenesis as reported previously in the case of certain infinite coordination polymers. ${ }^{18}$ A time-dependent analysis of the gel formation mechanism indicates that, at the initial stages of development, globular seeds are formed (Fig. 2b) which agglomerate to form linear as well as branched a

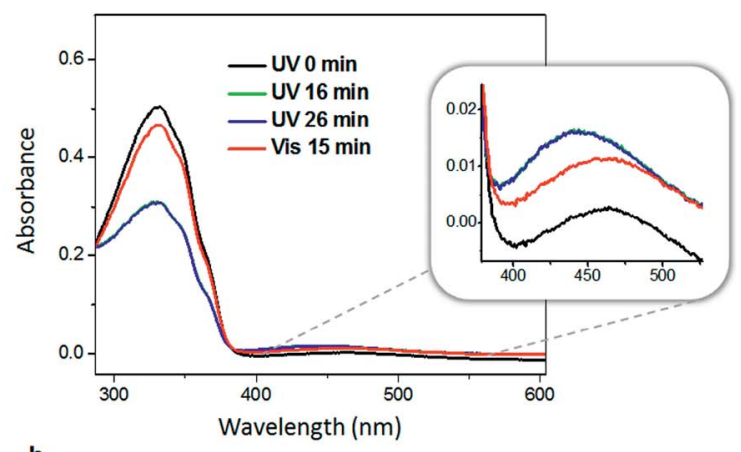

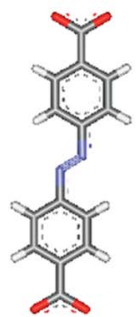

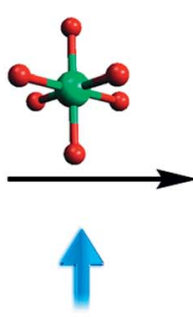

$\mathrm{Fe}\left(\mathrm{NO}_{3}\right)_{3} \cdot 9 \mathrm{H}_{2} \mathrm{O}$ solution is added subsequently

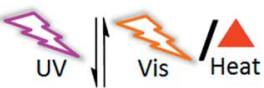

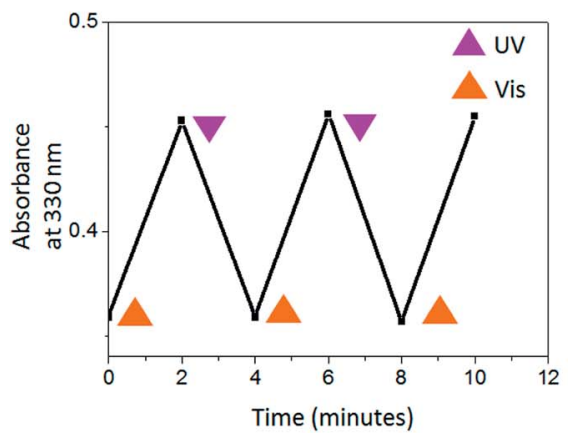
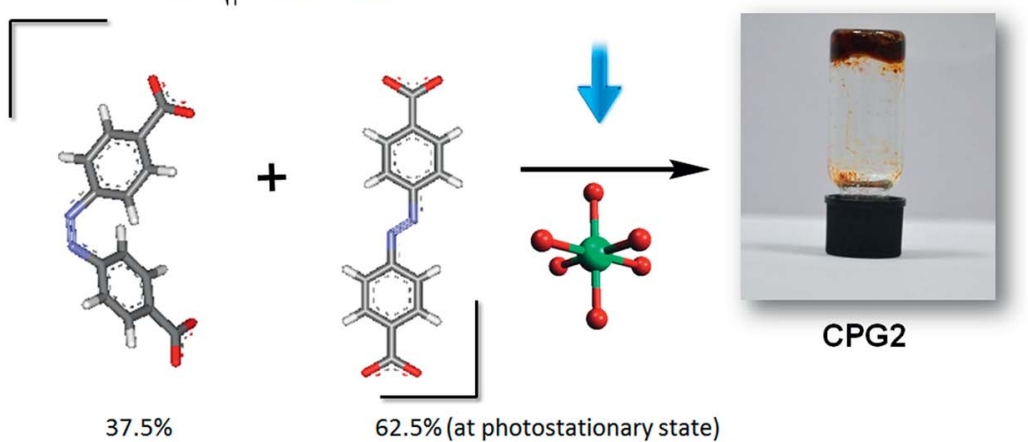

$62.5 \%$ (at photostationary state)

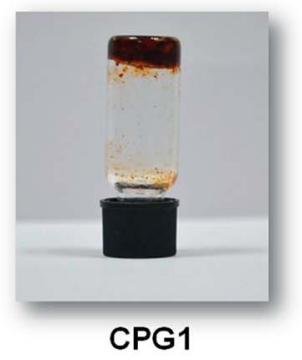

CPG1

Fig. 1 (a) Absorption spectra of the ADA solution in DMSO $\left(1.85 \times 10^{-5} \mathrm{M}\right)$ before and after irradiation with ultraviolet (UV) and visible (Vis) light. Inset shows zoomed region of $379-527 \mathrm{~nm}$ corresponding to the $n-\pi *$ transition band of cis azobenzene. (b) Reversibility of photoisomerisation, shown for three cycles. (c) Schematic representation of synthesis of CPG1 and CPG2 (corresponding gel images are shown). Trans and cis isomers of ADA (carbon - grey; nitrogen - blue; oxygen - red; hydrogen - white) and probable structure of inorganic octahedra (iron - green; oxygen - red) are also shown. 

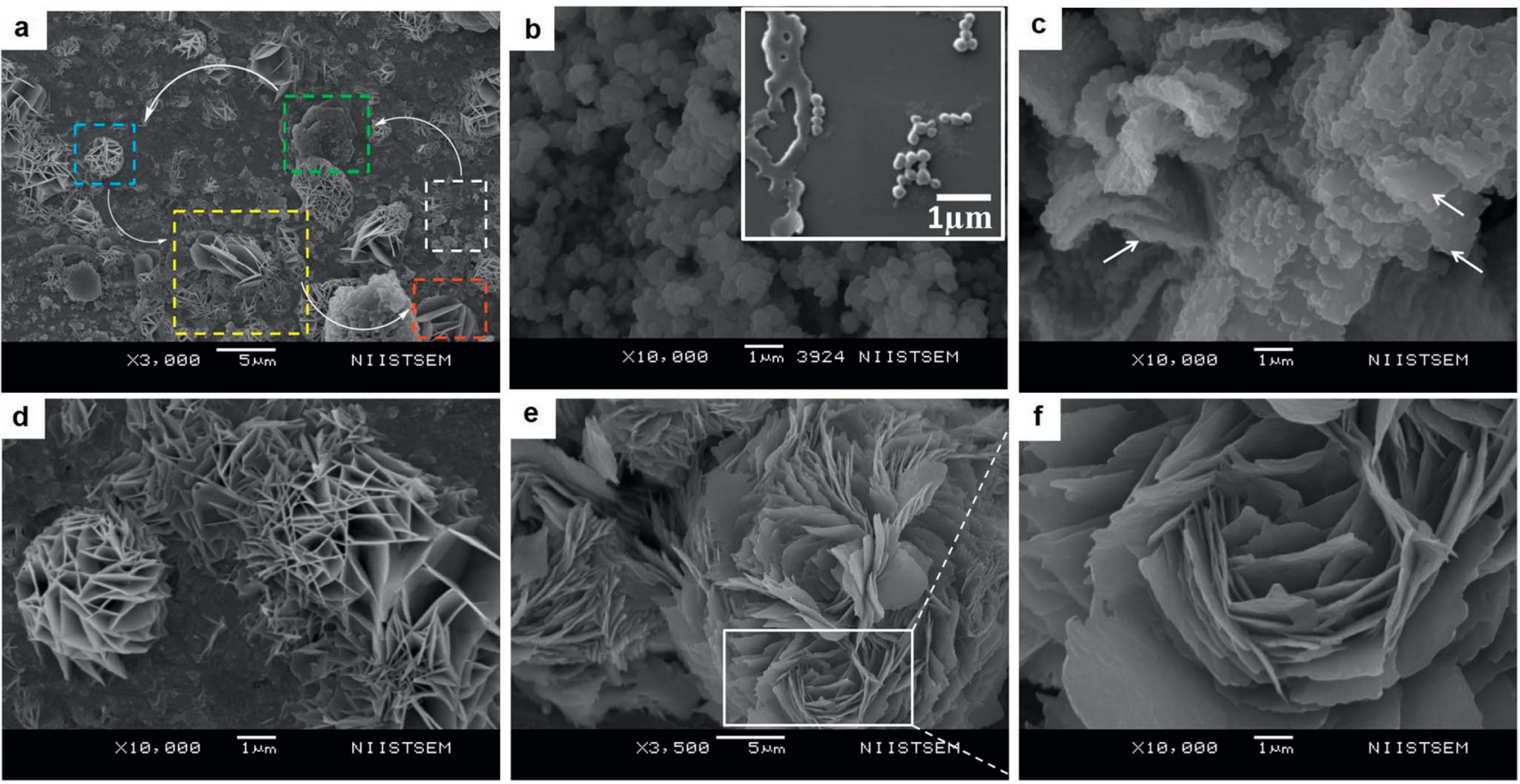

Fig. 2 SEM analysis of CPG1. (a) Different morphological forms of CPG1 captured in one frame. Platelets (white box), rough sheets (green box), cabbage (blue box), large smooth sheets (yellow box) and flower (red box) like structures. (b-f) Time dependent SEM analysis of CPG1. (b) Nanoscale CPG1 platelets formed immediately, inset shows platelets undergoing fusion. (c and d) Images obtained after 5-6 h. (c) Gradual smoothening of rough sheets; smoothened portions marked with arrows. (d) Formation of cabbage like structure. (e) Matured metal-organic flowers formed after 1 day. (f) Zoomed view of the box marked in (e), showing nanoscale sheets $(\sim 25 \mathrm{~nm})$.
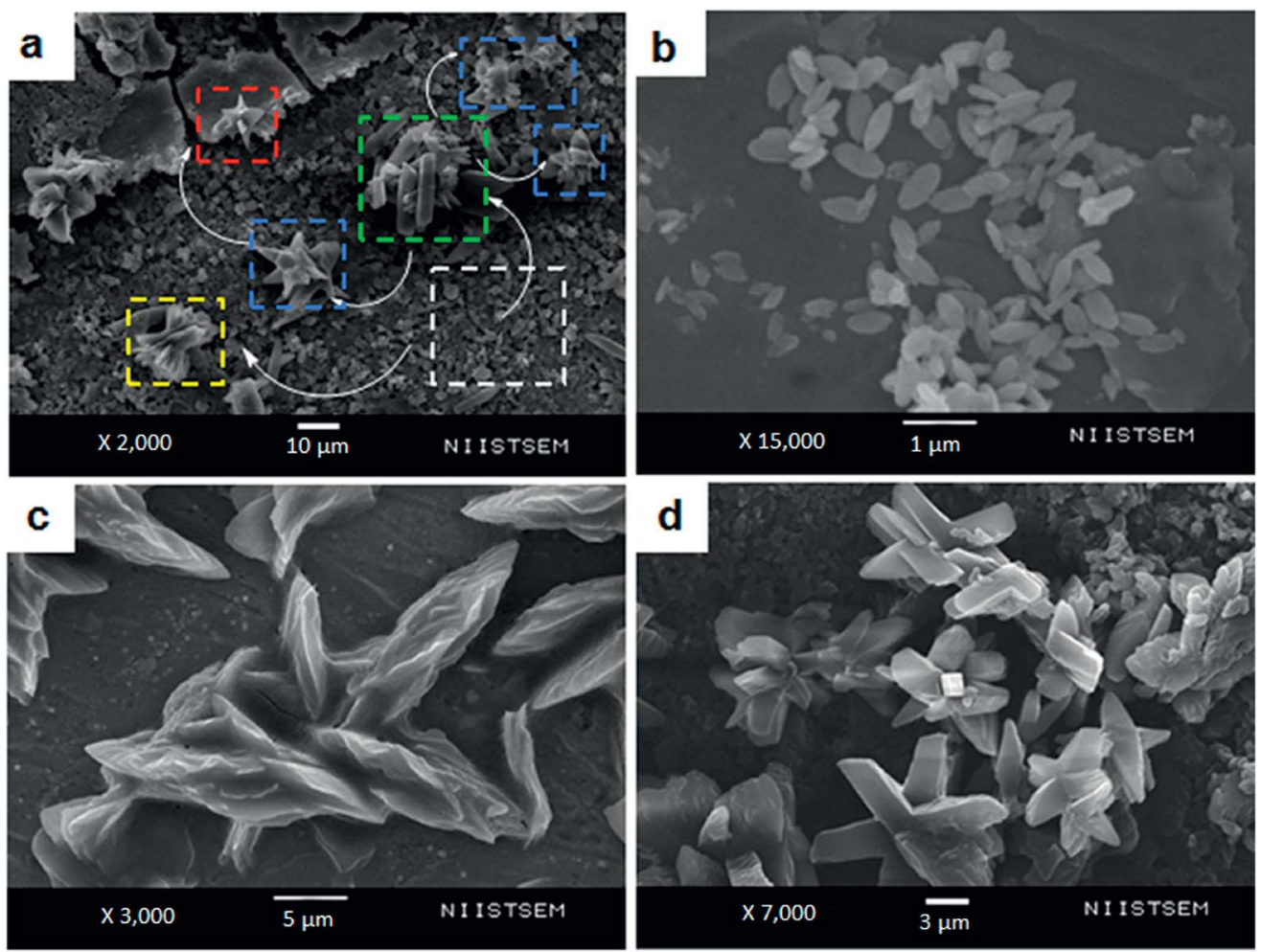

Fig. 3 SEM analysis of CPG2. (a) Different morphological forms of CPG2 captured in one frame. Leaves (white box), thick leaves (yellow box), tentacles (green box), stars in the formation (blue box), and crystalline stars (red box). (b-d) Time dependent SEM analysis of CPG2. (b) Nanoscale leaves of CPG2 after immediate gelation. (c) Aggregation of nano leaves after $1 \mathrm{~h}$. (d) Crystalline metal-organic stars obtained after 1 day. 

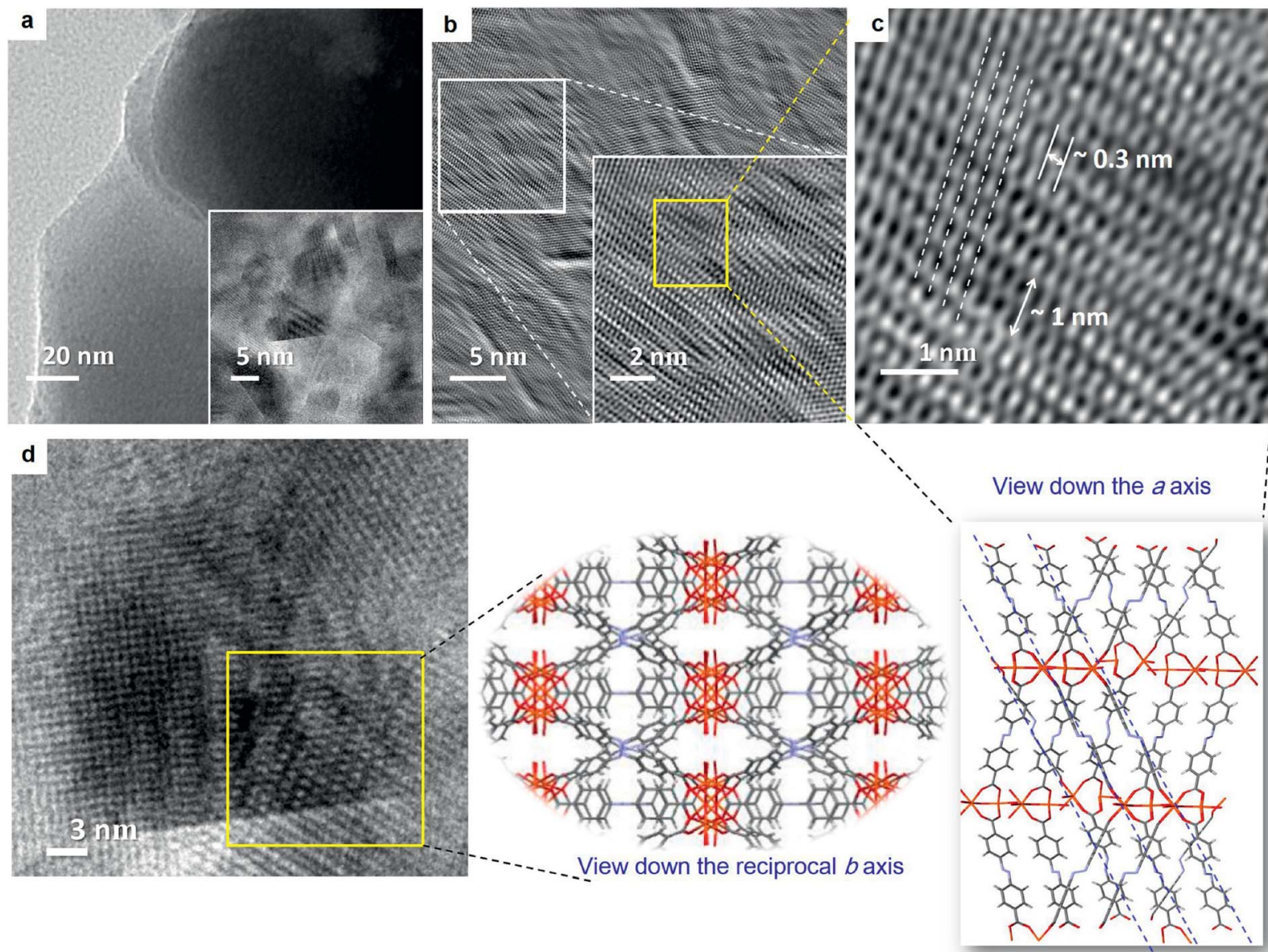

Fig. 4 (a) TEM image of CPG1 nanosheets. HRTEM image of a nanosheet is shown in the inset. (b) IFFT reconstruction of the HR-TEM image shown as inset in (a). Zoomed view of the marked box region is shown as inset. (c and d) Molecular packing of the coordination polymeric chains observed from the reconstructed HR-TEM image. Crystal structure viewed down axis ' $a$ ' and reciprocal cell axis ' $b$ ' of PCN $243,{ }^{26 b}$ are shown as a guide to the eye.

aggregates that grow into two-dimensional (2-D) sheets. However, the surface of larger sheets initially have a rough topography, which got smoothed by a gradual process of annealing (Fig. 2c). Folding of the smaller sheets lead to a cabbage like structure (Fig. 2d) followed by the sideways stacking of the sheets, which ultimately lead to metal-organic flowers (Fig. 2e). These micrometer sized flowers had nanoscale features in which the sheets were found to be about $25 \mathrm{~nm}$ thick (Fig. 2f). Transmission electron microscopy (TEM) analysis of CPG1 also confirmed a sheet like morphology. Lattice planes were clearly visible in the high-resolution transmission electron microscopy (HR-TEM) images, suggesting a highly ordered structure (Fig. 4a, inset).

In order to check the photoresponsive nature, we irradiated the gel sample with UV light. However, no noticeable change in morphology or properties was observed, and the gel remained intact even after hours of UV irradiation. Therefore, we thought of exploring the effect of the photoisomerisation of ADA before the preparation of the gel. The absorption spectrum of ADA in DMSO $\left(1.85 \times 10^{-5} \mathrm{M}\right)$, showed two bands with maxima at 330 and $440 \mathrm{~nm}$, corresponding to $\pi-\pi^{*}$ and $\mathrm{n}-\pi^{*}$ transitions, respectively (Fig. 1a). Photoisomerisation studies revealed that the molecule reaches a photostationary state within $16 \mathrm{~min}$, where $\sim 37.5 \%$ of the molecules are present in the cis form (Fig. 1a). The photoisomerisation of the azobenzene core was clearly reversible with respect to UV and visible light $\left(\lambda_{\text {bandpass }}=\right.$ 350 and $420 \mathrm{~nm}$, LOT-Oriel $200 \mathrm{~W}$ high-pressure Hg Lamp) and was also reproducible over several repeated cycles (Fig. 1b). Thus, when a solution of ADA and triethylamine in DMSO $\left(20 \mathrm{mg} \mathrm{mL}^{-1}\right)$ was irradiated with UV light $\left(\lambda_{\text {bandpass }}=350 \mathrm{~nm}\right)$ for about $4 \mathrm{~h}$ (to confirm that maximum conversion has taken place) and then a solution of $\mathrm{Fe}\left(\mathrm{NO}_{3}\right)_{3} \cdot 9 \mathrm{H}_{2} \mathrm{O}$ in DMSO was added to it, the solution mixture slowly turned into a gelatinous material, which was kept under UV light for about an hour and then analysed. The morphology of the irradiated metal-organic gel (CPG2) was quite different (Fig. 3) from its non-irradiated counterpart, CPG1 (Fig. 2). The morphology of CPG2 comprised of six armed metal-organic stars. Akin to the growth process of CPG1, CPG2 also showed a gradually evolving process starting from small leaf-like seeds (Fig. 3b), which grew with time to form thick leaves and tentacles (Fig. 3c) and ultimately evolved into star-like superstructures that possessed well-defined facets 


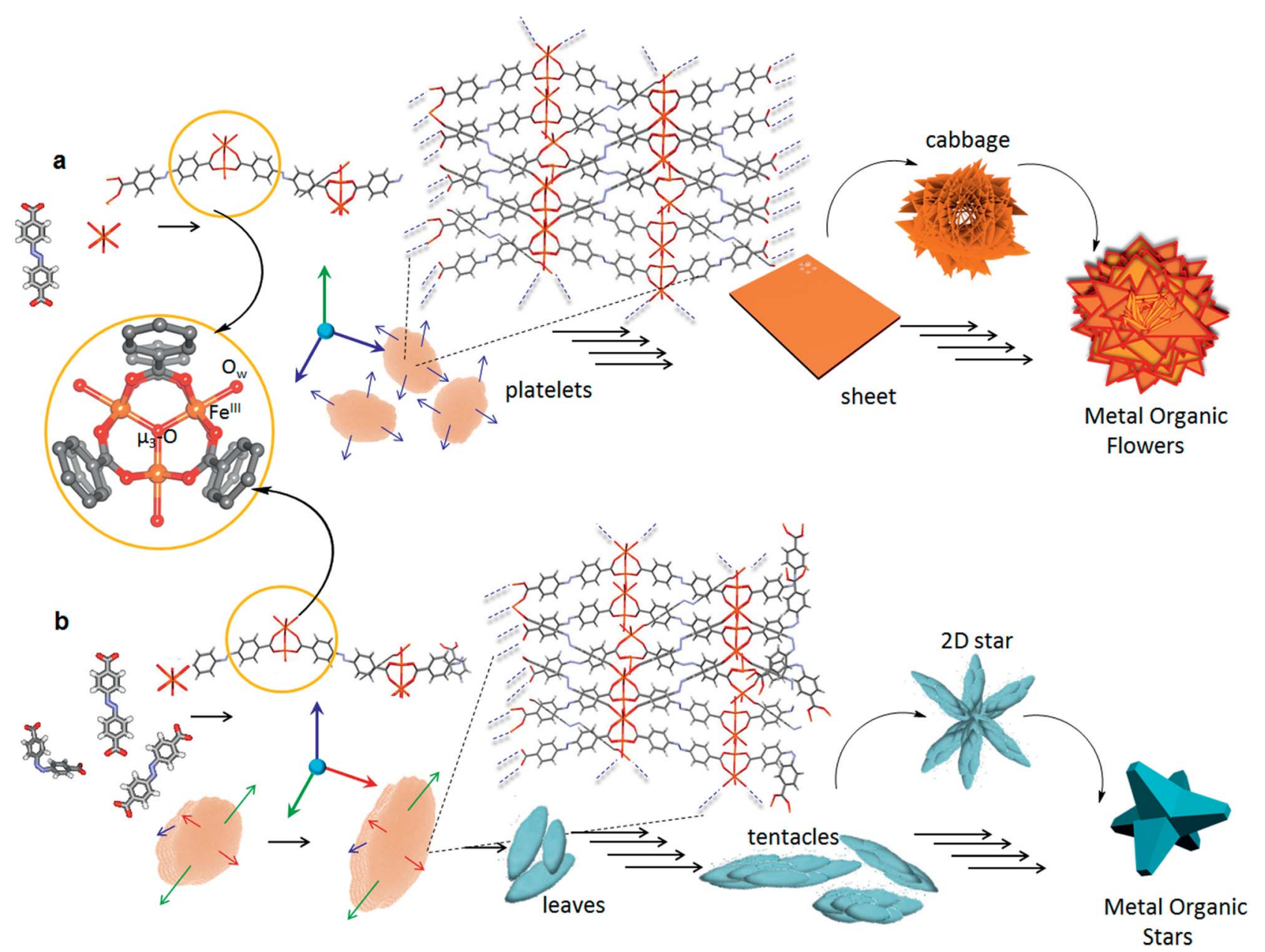

Fig. 5 Proposed formation pathways of (a) flowers in CPG1 and (b) stars in CPG2. Detailed view of the secondary building unit (SBU) containing the $\left[\mathrm{Fe}_{3}\left(\mu_{3}-\mathrm{O}\right)(\mathrm{COO})_{6}\right]$ cluster with coordinated solvent molecules $(\mathrm{OW})$. Each Fe ${ }^{\text {III }}$ center is located in a highly distorted octahedral environment. Fusion of platelets in CPG1 leads to the formation of 2-D sheets and finally into flowers. The anisotropic growth along different facets in CPG2 leads to the formation of stars. Blue, green and red arrows represent maximum, moderate and restricted rates of crystal growth. Blue broken lines indicate directions of polymeric extension. Not all short contacts are shown so as to retain visual clarity. A different crystallographic view of PCN $243,{ }^{26 b}$ can be found in Fig. S8.†

as that of a crystal (Fig. 3d). The transformation of tentacles to stars may be via a 2-D star-like architecture as found in several places in the SEM images (Fig. S2 $\dagger$ ). The presence of nano leaves and tentacles were confirmed from TEM analysis (Fig. S3 $\dagger$ ).

\section{Analysis of growth process of CPG1 and CPG2}

Although in time-dependent experiments we could mostly observe a particular morphology after a certain time interval, more than one morphological form were also found (Fig. 2a and $3 \mathrm{a})$ at a few places in both the samples, which suggest that the growth process was not only time dependent but also depends on the local concentration of the initial components. Most of the intermediate structures formed might be controlled by kinetic processes rather than thermodynamic control and are therefore limited by diffusion (Fig. $\mathrm{S} 4 \dagger$ ). ${ }^{19}$ At lower concentration of particles, the process of aggregation is controlled by diffusion leading to the formation of a patterned structure with random branching, whereas higher concentration leads to fast aggregation process leading to the formation of more compact structures. This process happens due to the large surface to volume ratio and high collision frequency of the particles. The occurrence of several unreacted sites on the surface of the seeds due to fast nucleation process, results in further polymerisation through coordination linkages and thereby fusion of the particles. With the gradual decrease in the concentration of the ligands and the metal ions, the nucleation of new particles is reduced and annealing of existing nuclei prevails much like the Ostwald ripening process. ${ }^{5 b}$ Unlike a single step thermodynamically controlled pathway, a kinetically controlled crystal growth process usually takes place via a sequence of steps involving amorphous intermediates. Such transformations can be easily tuned by various chemical stimuli (additives), which influence the energy of activation of nucleation (n), growth (g) and phase transformation $(\mathrm{t})$ processes. ${ }^{20}$ Photoirradiation of trans-ADA molecules with UV light leads to the formation of energetically unstable cis-ADA isomer, which in combination 

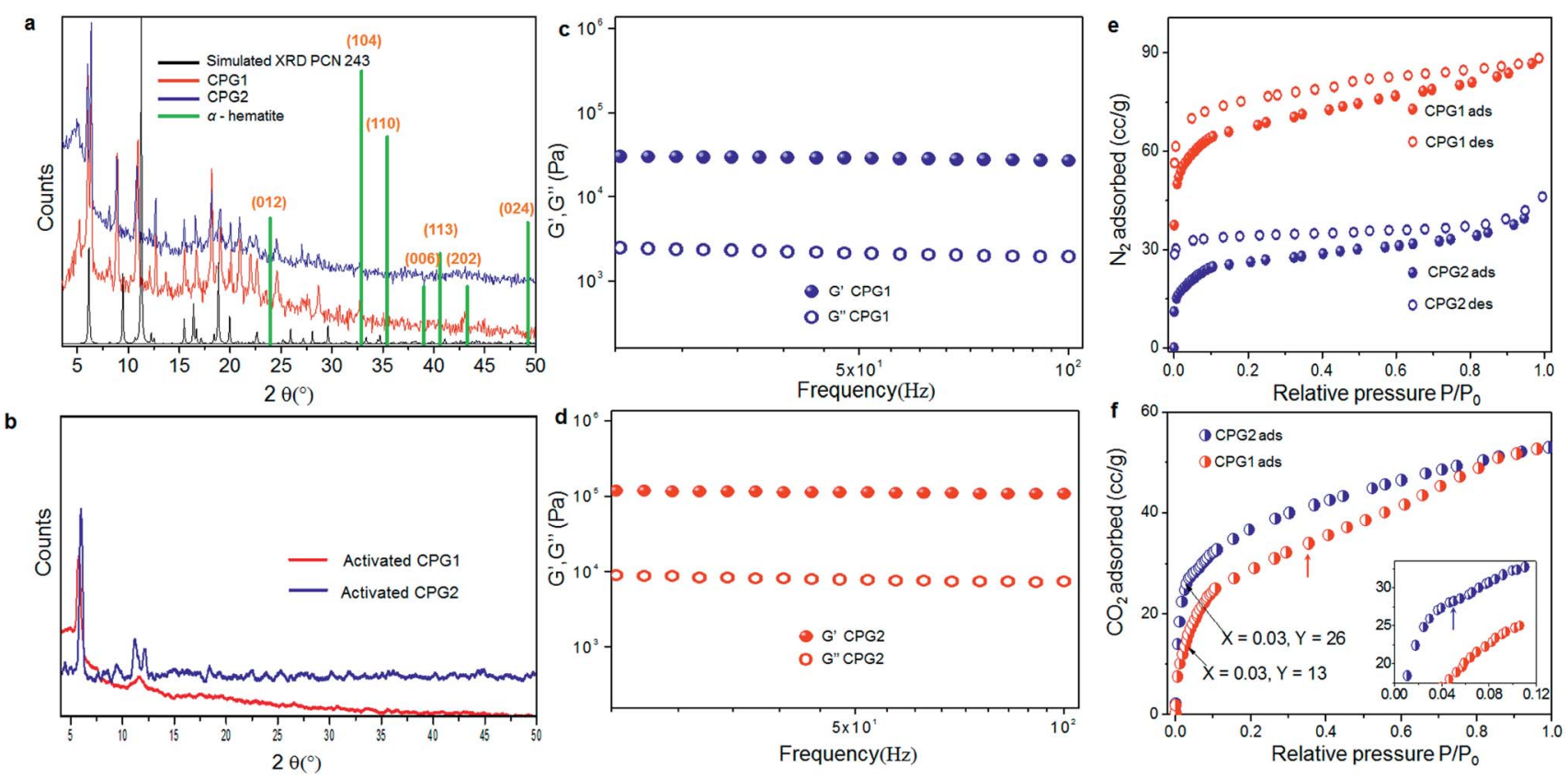

Fig. 6 (a) XRD pattern of CPG1 and CPG2. Simulated XRD pattern of PCN 243 and stick pattern of rhombohedral hematite $\left(\alpha-\mathrm{Fe}_{2} \mathrm{O}_{3}, \mathrm{JCPDS}\right.$ card no. 24-0072) phase also shown for comparison. (b) XRD pattern of CPG1 and CPG2 activated at $150{ }^{\circ} \mathrm{C}$. Rheological behaviour of (c) CPG1 and (d) CPG2. (e) Comparative $\mathrm{N}_{2}$ adsorption (ads) and desorption (des) isotherms of CPG1 and CPG2 at $77 \mathrm{~K}$. (f) Comparative $\mathrm{CO}_{2}$ adsorption isotherms of CPG1 and CPG2 at $195 \mathrm{~K}$. Inset shows the zoomed low-pressure region. Red and blue arrows indicate stepwise uptake of $\mathrm{CO}_{2}$ for $\mathrm{CPG}$ and CPG2, respectively.

with trans-ADA molecules can significantly influence the nucleation-growth-transformation processes. Therefore, a new kinetic pathway is followed, which is shown in a modified energy profile diagram as proposed previously by Mann and co-workers (Fig. S5†). ${ }^{20 a}$ In order to confirm our hypothesis, we have carried out the synthesis of nanoscale CPG1 and CPG2 under thermodynamic conditions at a very low concentration where we were able to arrive at only nano platelets and leaves (Fig. S6†).

According to the Bravais, Friedel, Donnay and Harker (BFDH) law, the morphology of a crystal is mainly determined by the slowest growing facet of the crystal. ${ }^{21}$ The 2-D nucleus on a particular crystal facet determines the overall crystal morphology depending on the probabilities of growth or dissolution at a particular site. A crystal in principle should grow in a particular direction unless the energetics is changed along a particular facet. Now, taking a close look at the formation mechanism (Fig. S7 $\dagger$ ) for both CPG1 and CPG2, one can assume that the transformation of platelets (for CPG1) to leaves (for CPG2) essentially occurs by a similar process of anisotropic crystal growth. At the photostationary state $\sim 37.5 \%$ of cis-ADA can trigger this anisotropic growth mechanism by varying the crystal growth rates along different facets. It is known that the cis isomer of azobenzene can form energetically less favourable self-assembled structures. ${ }^{22}$ In case of a coordination bond driven self-assembly, the non-planar nature of cis-ADA can further hamper the chain building process as the geometry of the inorganic cluster brings cis-ADA closer to the coordination polymeric chain, where it remains in a sterically unfavourable position to undergo further reaction. Thus, cis-ADA can essentially act as a capping agent, which stops the crystal growth along a particular facet. The presence of these capping agents is difficult to verify even experimentally, and therefore, the explanation of such process mainly relies on speculation, in the absence of a completely understood crystal growth mechanism..$^{23}$ It is hypothesised that the rate of crystal growth may range from moderate to maximum along different facets (Fig. 5). This anisotropy arises only after smaller fragments (oligomers) of the coordination polymeric chain, containing trans-ADA $(\sim 62.5 \%)$ nucleate to form platelet like structures at the threshold of nanoscale. Transformation of platelets to leaves essentially takes place at the mesoscale followed by aggregation of preformed crystalline nano-leaves leading to the formation of microscopic superstructures. While trying to understand the growth process in these materials, it came to our notice that the morphology of colloidal hematite, a naturally occurring mineral, is also affected by similar aggregation mediated crystal growth process, showcasing several polymorphs ranging from platelets, ellipsoidal particles to stars. One can have a chemical control over the growth of different facets to facilitate such anisotropic crystal growth. ${ }^{24}$ The same control has been achieved in the present case via photonic manipulation. A number of reports towards engineering the self-assembly process of framework materials at the mesoscopic domain are known. ${ }^{25}$ It is important to note that in all these cases, a third component, for instance, a coordination modulator or a template was necessary to achieve such mesoscopic control over self-assembly. Whereas, in the present case, a 
certain fraction of the isomerised organic ligand is enough to bifurcate the bottom-up self-assembly process, while other cis-ADA molecules may get entrapped in the microporous structure. The cis-ADA molecules can gradually reconvert back to the thermodynamically more stable trans-ADA. The fact that cis-ADA reconverted to trans-ADA in CPG2 was confirmed from the FT-IR studies where no new peak at higher wavenumber region $\left(\sim 720 \mathrm{~cm}^{-1}\right)$ corresponding to cis-ADA was observed. ${ }^{6 c}$ The peak corresponding to the trans isomer $\left(700 \mathrm{~cm}^{-1}\right)$ in free ADA was shifted to $709 \mathrm{~cm}^{-1}$ in CPG1 and observed as a shoulder in CPG2 along with a prominent peak at $700 \mathrm{~cm}^{-1}$ corresponding to free trans-ADA. This was further confirmed from the deconvoluted FT-IR spectra of CPG2, where the peaks at 1688,1424 and $1294 \mathrm{~cm}^{-1}$ corresponding to free ADA, which were absent in CPG1, were found to be present in CPG2 (Fig. S1†). The results also indicate the shifting of the asymmetric stretching frequency of ADA towards the lower wavenumber region, basically indicating that the bidentate coordination modes become more prominent in CPG2.

One of the problems of working with $\mathrm{Fe}(\mathrm{III})$ or $\mathrm{Al}(\mathrm{III})$ (hard acid) carboxylate (hard base) coordination polymers is the immediate formation of strong metal-ligand coordination, that does not allow defect rectification during the crystal growth process, leading towards precipitates or gels in most cases. ${ }^{26}$ The in situ formation of multiple structure directing inorganic building blocks under kinetic conditions often adds to this problem. ${ }^{16 a, b, 26 b}$ Therefore, the actual structure determination in the gel state, containing a mixture of polymorphic structures, is difficult to assign, even via computational simulation. However, we can derive some idea from inverse fast Fourier transform (IFFT) reconstructed HR-TEM images, which showed the existence of coordination polymeric chains arranged in a parallel manner (Fig. $4 \mathrm{~b}$ and c). This image matches with the recently reported crystal structure of PCN-243, ${ }^{26 b}$ a MOF formed from ADA and an $\mathrm{Fe}(\mathrm{III})$ based inorganic cluster, when viewed along the $a$-axis. The distance between the polymeric chains roughly corresponds to $\sim 0.3 \mathrm{~nm}$. Further, the presence of ADA (molecular length $\sim 1.12 \mathrm{~nm}$ ) in the polymeric chains can be understood from the slightly kinked structure of the azobenzene core. Similarly, the rhombic pores visualised in Fig. $4 \mathrm{~d}$ can be compared with the same crystal structure, if viewed along the $b$-axis. Elemental analysis, energy dispersive X-ray (EDX) analysis and thermogravimetric analysis (TGA) of the two forms showed similar composition, solvent weight loss and thermal decomposition pattern (Fig. S9 and S10 $†$ ). The powder X-ray diffraction (XRD) pattern of the two forms CPG1 and CPG2 also did not show considerable difference (Fig. 6a) pointing that both the morphological form possess similar metal-linker connectivity. The simulated XRD pattern obtained from PCN-243 matches well with the observed pattern in CPG1 and CPG2 although the possibility of other amorphous phases cannot be completely ruled out, as evident from the presence of a broad halo $\left(2 \theta 12-30^{\circ}\right)$ in the XRD pattern of both the materials. However, the XRD peaks corresponding to hematite $\left(\alpha-\mathrm{Fe}_{2} \mathrm{O}_{3}\right)$ phase with rhombohedral structure $(a, b=0.5 \mathrm{~nm}, c=$ $1.38 \mathrm{~nm}$, JCPDS card no. 24-0072) is completely absent in either CPG1 or CPG2 (Fig. 6a). Also the FT-IR peaks corresponding to the lattice vibrations in $\alpha-\mathrm{Fe}_{2} \mathrm{O}_{3}\left(\sim 500-600 \mathrm{~cm}^{-1}\right)$ is absent in either case. ${ }^{24 b}$ These data confirm that the observed unusual splitting of the growth process in our material was not affected by pseudomorphic growth replication process over hematite or presence of hematite phase as an impurity (Fig. S1†). ${ }^{25 a}$ From the above investigations, we conclude that the strong hard acidhard base interactions results in fast unidentate coordination, and thereby gelation. With time, most of the amorphous intermediates are converted to more crystalline forms, which show bidentate coordination modes. Selected area electron diffraction (SAED) analysis of both CPG1 and CPG2 revealed multiple diffraction patterns ranging from amorphous, polycrystalline to single crystalline behavior (Fig. S11 $\dagger$ ). It is also observed that the presence of cis-ADA in the formation of CPG2 basically slows down the crystallisation process within the gel matrix resulting in more crystalline products. As a result, the presence of amorphous to highly crystalline species in the xerogel matrix makes the system highly heterogeneous for correlation of structure with properties.

Further, in order to confirm that the mesoscopic change in the morphology is only due to the photoresponsive moiety and not due to formation of different inorganic cluster formation under UV light irradiation, a similar system derived from a nonphotoisomerisable organic ligand, trimesic acid was subjected to UV light irradiation (Fig. S12†). In this case, we could not observe any change in the morphology, revealing that the inorganic cluster was not affected by any external physical stimulus such as light, unless it comes in contact with a photoresponsive organic material.

\section{Comparative rheological behaviour and porosity analysis}

Gel-like behaviour of CPG1 and CPG2 was confirmed from rheological studies where for both the materials, the value of elastic storage modulus $\left(G^{\prime}\right)$ was found to be invariant with the applied frequency and it also exceeded the value of elastic loss modulus $\left(G^{\prime \prime}\right)$ by an order of magnitude. These studies also lead to the conclusion that in comparison to CPG1, CPG2 has a greater storage modulus, and is more viscoelastic in nature (Fig. 6c and d). The XRD pattern of both the gels activated prior to gas adsorption studies when compared with that of the as-synthesised form indicate a lesser degree of transformation from the pristine crystalline form for activated CPG2, while activation of CPG1 transformed it almost into an amorphous material (Fig. 6b). Thus, application of heat or stress leads to less change in structure in the case of CPG2, corroborating greater rigidity and ability to retain its parent structure as compared to CPG1. This rigidity may be attributed to the compact packing of the molecular motifs in CPG2 as compared to CPG1.

In order to have an idea about the porosity of the two materials, $\mathrm{N}_{2}$ gas adsorption measurements were performed on both CPG1 and CPG2. The adsorption isotherm obtained in both cases showed a typical type I behaviour according to International Union of Pure and Applied Chemistry (IUPAC) classification, confirming microporosity of the samples. ${ }^{6 g, 27}$ Significant hysteresis was observed in the process of desorption 
for both samples. The micropore dimensions calculated from the pore size distribution plot (10.2 ̊) (Fig. S13†) matches well with the length of ADA. The maximum amount of $\mathrm{N}_{2}$ adsorbed at saturation pressure in the case of CPG1 was found to be 88 $\mathrm{mL} \mathrm{g}^{-1}$ and it possessed a Brunauer-Emmett-Teller (BET) surface area $\sim 223 \mathrm{~m}^{2} \mathrm{~g}^{-1}$. In the case of CPG2, maximum uptake was found to be $46 \mathrm{~mL} \mathrm{~g}^{-1}$ of the sample and the BET surface area was substantially decreased to $\sim 90 \mathrm{~m}^{2} \mathrm{~g}^{-1}$ (Fig. 6e). The difference in gas adsorption properties has been compared with previous literature reports, where $\mathrm{N}_{2}$ and $\mathrm{CO}_{2}$ adsorption has been measured as a function of morphology or photoresponsive behaviour, and results are summarised in Tables S1 and $\mathrm{S} 2, \uparrow$ which indicate that the porosity difference obtained in our material is quite significant. Apart from this, the surface area is higher when compared to previously reported xerogels and aerogels, ${ }^{28}$ although aerogels obtained by supercritical $\mathrm{CO}_{2}$ treatment are known to exhibit better porosity in most cases. ${ }^{\mathbf{1 6}}$ In the absence of a particular crystalline phase, the drop-off in $\mathrm{N}_{2}$ gas uptake in CPG2 may be ascribed to the loss of surface area due to downsizing of the coordination network combined with considerable amount of pore blocking due to the presence of encapsulated ADA molecules, as evidenced from the FT-IR studies. As a result, CPG2 possess lower available free volume due to considerable pore blocking. The presence of encapsulated ADA molecules, leading towards a more close packed structure, supports the improvement in rheological behaviour. However, the $\mathrm{CO}_{2}$ adsorption isotherm (Fig. 6f) showed that at low partial pressure (0.03), CPG2 adsorbs almost double the amount of $\mathrm{CO}_{2}$ than CPG1. This comparatively high uptake of $\mathrm{CO}_{2}$ at lower pressure can be assigned to the ability of CPG2 to undergo a spatial adjustment to expose the lone pairs of electrons over nitrogen atoms towards the pores, ${ }^{29}$ which can then act as potential sites for $\mathrm{CO}_{2}$ adsorption (Fig. $6 \mathrm{f}$, inset). ${ }^{30} \mathrm{CPG} 1$ on the other hand shows a broad second step in the adsorption isotherm at higher partial pressure $(0.46)$ to finally match the total amount of adsorbed $\mathrm{CO}_{2}$ found in the case of CPG2. This can be ascribed to the guest-induced second-order structural transformations taking place in CPG1. ${ }^{25 b}$ At higher pressures, CPG2 did not show any such change, indicating its densely packed rigid structure.

\section{Conclusions}

Results of this study point toward the conclusion that, irrespective of the presence of a photoresponsive group in CPG1 or CPG2, light does not have a post-synthetic influence on its bulk structure (Fig. S14 $\dagger$ ). However, pre-synthetic photoisomerisation of the precursor of the coordination polymer leads to morphologically different macroscopic structures. Time-dependent morphological investigations suggest that light-induced structural evolution nucleates at the molecular level, transforms at the mesoscopic level via controlled facetted crystal growth process, leading to different macroscopic morphological forms of CPG2 in the present case. Photoinduced coordination modulation can therefore be applied as a promising tool for 'nanoarchitectonics', to control the kinetic self-assembly of photoresponsive coordination polymers. It is further observed that the aforesaid synthetic strategy can have a profound influence on the material properties of porous metalorganic materials. Such porous gels like CPG2 with better rheological properties along with comparatively lower intake of $\mathrm{N}_{2}$ and better $\mathrm{CO}_{2}$ capture properties can easily replace the pore blocking binder materials used in generating shaped bodies from MOFs for industrial applications. ${ }^{31}$ Also the generation of iron oxide nanoparticles from such gel templates would be of interest for further investigation. ${ }^{32}$

\section{Acknowledgements}

We thank the Council of Scientific and Industrial Research (CSIR), Government of India for financial support. A.A. is grateful to Department of Atomic Energy for a DAE-SRC Outstanding Researcher award. R.D.M. is thankful to CSIR, Government of India for a research fellowship. Dr J. D. Sudha, Mr C. K. Chandrakanth and Mr Kiran Mohan are acknowledged for rheology, SEM and TEM experiments, respectively.

\section{Notes and references}

1 (a) M. Aono, Sci. Technol. Adv. Mater., 2011, 12, 040301; (b) K. Ariga, Q. Ji, J. P. Hill, Y. Bando and M. Aono, NPG Asia Mater., 2012, 4, e17; (c) K. Ariga, Q. Ji, W. Nakanishi, J. P. Hill and M. Aono, Mater. Horiz., 2015, 2, 406; (d) K. Ariga, Y. Yamauchi, G. Rydzek, Q. Ji, Y. Yonamine, K. C.-W. Wu and J. P. Hill, Chem. Lett., 2014, 43, 36.

2 (a) H. Li, M. Eddaoudi, M. O'Keeffe and O. M. Yaghi, Nature, 1999, 402, 276; (b) H. Furukawa, K. E. Cordova, M. O'Keeffe and O. M. Yaghi, Science, 2013, 341, 1230444.

3 (a) M. Kondo, T. Yoshitomi, K. Seki, H. Matsuzaka and S. Kitagawa, Angew. Chem., Int. Ed. Engl., 1997, 36, 1725; (b) S. Kitagawa, R. Kitaura and S. Noro, Angew. Chem., Int. Ed., 2004, 43, 2334.

4 (a) N. Kishi, M. Akita, M. Kamiya, S. Hayashi, H.-F. Hsu and M. Yoshizawa, J. Am. Chem. Soc., 2013, 135, 12976; (b) R. D. Mukhopadhyay, V. K. Praveen and A. Ajayaghosh, Mater. Horiz., 2014, 1, 572.

5 (a) A. Gopal, M. Hifsudheen, S. Furumi, M. Takeuchi and A. Ajayaghosh, Angew. Chem., Int. Ed., 2012, 51, 10505; (b) S. Mahesh, A. Gopal, R. Thirumalai and A. Ajayaghosh, J. Am. Chem. Soc., 2012, 134, 7227; (c) R. Rajaganesh, A. Gopal, T. M. Das and A. Ajayaghosh, Org. Lett., 2012, 14, 748; (d) G. Abellán, E. Coronado, C. Martí-Gastaldo, A. Ribera, J. L. Jordá and H. García, Adv. Mater., 2014, 26, 4156.

6 (a) A. Modrow, D. Zargarani, R. Herges and N. Stock, Dalton Trans., 2011, 40, 4217; (b) J. Park, D. Yuan, K. T. Pham, J. R. Li, A. Yakovenko and H. C. Zhou, J. Am. Chem. Soc., 2012, 134, 99; (c) N. Yanai, T. Uemura, M. Inoue, R. Matsuda, T. Fukushima, M. Tsujimoto, S. Isoda and S. Kitagawa, J. Am. Chem. Soc., 2012, 134, 4501; (d) R. Lyndon, K. Konstas, B. P. Ladewig, P. D. Southon, P. C. Kepert and M. R. Hill, Angew. Chem., Int. Ed., 2013, 52, 3695; (e) J. W. Brown, B. L. Henderson, M. D. Kiesz, A. C. Whalley, W. Morris, S. Grunder, H. Deng, 
H. Furukawa, J. I. Zink, J. F. Stoddart and O. M. Yaghi, Chem. Sci., 2013, 4, 2858; (f) L. Heinke, M. Cakici, M. Dommaschk, S. Grosjean, R. Herges, S. Brase and C. Woll, ACS Nano, 2014, 8, 1463; $(g)$ J. Park, L. B. Sun, Y. P. Chen, Z. Perry and H. C. Zhou, Angew. Chem., Int. Ed., 2014, 53, 5842.

7 (a) S. Yagai and A. Kitamura, Chem. Soc. Rev., 2008, 37, 1520; (b) X. Yang, G. Zhang and D. Zhang, J. Mater. Chem., 2012, 22, 38.

8 (a) H. A. Barnes, J. Non-Newtonian Fluid Mech., 1997, 70, 1; (b) P. Dastidar, Chem. Soc. Rev., 2008, 37, 2699; (c) M.-O. M. Piepenbrock, G. O. Llyod, N. Clarke and J. W. Steed, Chem. Rev., 2010, 110, 1960; (d) J. Zhang and C.-Y. Su, Coord. Chem. Rev., 2013, 257, 1373; (e) S. S. Babu, V. K. Praveen and A. Ajayaghosh, Chem. Rev., 2014, 114, 1973.

9 (a) T. F. A. de Greef and E. W. Meijer, Nature, 2008, 453, 171; (b) T. Aida, E. W. Meijer and S. I. Stupp, Science, 2012, 335, 813.

10 (a) J. Li, I. Cvrtila, M. Colomb-Delsuc, E. Otten and S. Otto, Chem.-Eur. J., 2014, 20, 15709; (b) S. Saha, G. Das, J. Thote and R. Banerjee, J. Am. Chem. Soc., 2014, 136, 14845; (c) H. B. Aiyappa, S. Saha, P. Wadge, R. Banerjee and S. Kurungot, Chem. Sci., 2015, 6, 603.

11 (a) O. M. Yaghi, G. Li and H. Li, Chem. Mater., 1997, 9, 1074; (b) J. A. Foster, M.-O. M. Piepenbrock, G. O. Llyod, N. Clarke, J. K. Howard and J. W. Steed, Nat. Chem., 2010, 2, 1037.

12 K. J. C. van Bommel, A. Friggeri and S. Shinkai, Angew. Chem., Int. Ed., 2003, 42, 980.

13 H. Yamaguchi, Y. Kobayashi, R. Kobayashi, Y. Takashima, A. Hashidzume and A. Harada, Nat. Commun., 2012, 3, 603. 14 S. C. Wei, M. Pan, K. Li, S. Wang, J. Zhang and C. Y. Su, Adv. Mater., 2014, 26, 2072.

15 (a) R. F. Service, Science, 2012, 335, 1167; (b) M. Nakatsuji, Biomater. Sci., 2013, 1, 9.

16 (a) C. Serre, F. Millange, S. Surble and G. Ferey, Angew. Chem., Int. Ed., 2004, 43, 6285; (b) T. R. Whitfield, X. Wang, L. Liu and A. J. Jacobson, Solid State Sci., 2005, 7, 1096; (c) J. Zhang and C.-Y. Su, Coord. Chem. Rev., 2013, 257, 1373; (d) Q. Wei and S. L. James, Chem. Commun., 2005, 1555; (e) M. R. Lohe, M. Rose and S. Kaskel, Chem. Commun., 2009, 6056.

17 (a) G. B. Deacon and R. J. Phillips, Coord. Chem. Rev., 1980, 33, 227; (b) T. Ishiwata, Y. Furukawa, K. Sugikawa, K. Kokado and K. Sada, J. Am. Chem. Soc., 2013, 135, 5427.

18 (a) M. Oh and C. A. Mirkin, Nature, 2005, 438, 651; (b) S. Jung and M. Oh, Angew. Chem., Int. Ed., 2008, 47, 2049; (c) Y. M. Jeon, G. S. Armatas, D. Kim, M. G. Kanatzidis and C. A. Mirkin, Small, 2009, 5, 46; (d) A. M. Spokoyny, D. Kim, A. Sumrein and C. A. Mirkin, Chem. Soc. Rev., 2009, 38, 1218.
19 (a) T. Witten and L. Sander, Phys. Rev. Lett., 1981, 47, 1400; (b) J. Ge, J. Lei and R. N. Zare, Nat. Nanotechnol., 2012, 7, 428; (c) J. Zeng and Y. Xia, Nat. Nanotechnol., 2012, 7, 415.

20 (a) H. Cölfen and S. Mann, Angew. Chem., Int. Ed., 2003, 42, 2350; (b) J.-L. Li and X.-Y. Liu, Adv. Funct. Mater., 2010, 20, 3196.

21 (a) J. D. H. Donnay and D. Harker, Am. Mineral., 1937, 22, 446; (b) A. Umemura, S. Diring, S. Furukawa, H. Uehara, T. Tsuruoka and S. Kitagawa, J. Am. Chem. Soc., 2011, 133, 15506.

22 F. Rakotondradany, M. A. Whitehead, A. M. Lebuis and H. F. Sleiman, Chem.-Eur. J., 2003, 9, 4771.

23 (a) W. Cho, H. J. Lee and M. Oh, J. Am. Chem. Soc., 2008, 130, 16943; (b) T. Tsuruoka, S. Furukawa, Y. Takashima, K. Yoshida, S. Isoda and S. Kitagawa, Angew. Chem., Int. Ed., 2009, 48, 4739.

24 (a) E. Matijević and P. Scheiner, J. Colloid Interface Sci., 1978, 63, 509; (b) M. Ocaña, M. P. Morales and C. J. Serna, J. Colloid Interface Sci., 1995, 171, 85.

25 (a) J. Reboul, S. Furukawa, N. Horike, M. Tsotsalas, K. Hirai, H. Uehara, M. Kondo, N. Louvain, O. Sakata and S. Kitagawa, Nat. Mater., 2012, 11, 717; (b) Y. Sakata, S. Furukawa, M. Kondo, K. Hirai, N. Horike, Y. Takashima, H. Uehara, N. Louvain, M. Meilikhov, T. Tsuruoka, S. Isoda, W. Kosaka, O. Sakata and S. Kitagawa, Science, 2013, 339, 193.

26 (a) L. Li, S. Xiang, S. Cao, J. Zhang, G. Ouyang, L. Chen and C.-Y. Su, Nat. Commun., 2013, 4, 1774; (b) D. Feng, K. Wang, Z. Wei, Y. P. Chen, C. M. Simon, R. K. Arvapally, R. L. Martin, M. Bosch, T. F. Liu, S. Fordham, D. Yuan, M. A. Omary, M. Haranczyk, B. Smit and H. C. Zhou, Nat. Commun., 2014, 5, 5723.

27 (a) H. Sato, R. Matsuda, K. Sugimoto, M. Takata and S. Kitagawa, Nat. Mater., 2010, 9, 661; (b) W. Morris, C. J. Doonan and O. M. Yaghi, Inorg. Chem., 2011, 50, 6853. 28 (a) Y.-O. Liu, L. He, J. Zhang, X. Wang and C.-Y. Su, Chem. Mater., 2009, 21, 557; (b) J. Zhang, X. Wang, L. He, L. Chen, C.-Y. Su and S. L. James, New J. Chem., 2009, 33, 1070; (c) S. Samai and K. Biradha, Chem. Mater., 2012, 24, 1165.

29 Y. Takashima, V. M. Martinez, S. Furukawa, M. Kondo, S. Shimomura, H. Uehara, M. Nakahama, K. Sugimoto and S. Kitagawa, Nat. Commun., 2011, 2, 168.

30 C. M. Nagaraja, R. Haldar, T. K. Maji and C. N. R. Rao, Cryst. Growth Des., 2012, 12, 975.

31 (a) A. U. Czaja, N. Trukhan and U. Muller, Chem. Soc. Rev., 2009, 38, 1284; (b) S. Mitchell, N. L. Michels, K. Kunze and J. Perez-Ramirez, Nat. Chem., 2012, 4, 825.

32 (a) S. Alam, C. Anand, K. Ariga, T. Mori and A. Vinu, Angew. Chem., Int. Ed., 2009, 48, 7358; (b) W. Cho, S. Park and M. Oh, Chem. Commun., 2011, 47, 4138. 